\title{
Forebrain patterning defects in Small eye mutant mice
}

\author{
Anastassia Stoykova, Rüdiger Fritsch, Claudia Walther and Peter Gruss* \\ Max-Planck-Institute of Biophysical Chemistry, Am Fassberg, D-37077 Göttingen, Germany \\ *Author for correspondence (e-mail: pgruss@gwdg.de)
}

\section{SUMMARY}

Pax6 is a member of the Pax gene family of transcriptional regulators that exhibits a restricted spatiotemporal expression in the developing central nervous system, eye and nose. Mutations in Pax6 are responsible for inherited malformations in man, rat and mouse. To evaluate the role of Pax6 in forebrain development, we studied in detail mouse Small eye/Pax6 mutant brains. This analysis revealed severe defects in forebrain regions where Pax6 is specifically expressed. The establishment of some expression boundaries along the dorsoventral axis of the secondary prosencephalon is distorted and the specification of several ventral structures and nuclei is abolished. Specifically, the development of the hypothalamo-telencephalic transition zone and the ventral thalamus is distorted. Our detailed analysis included a comparison of the expression of Pax6, Dlx1 and several other genes during embryonic mouse brain development in wild-type and in the mutant Small eye (Sey) brain. The results from the analysis of normal brain development show that the restricted expression of Pax6 and DlxI at E12.5 dpc respect domains within the forebrain, consistent with the implications of the prosomeric model for the organisation of the forebrain (L. Puelles and J. L. R. Rubenstein (1993) Trends Neurosci. 16, 472-479). Furthermore, we found an early restriction of Pax6 and Dlxl expression into presumptive histogenetic fields that correlate with the formation of distinct forebrain structures and nuclei. Our results are discussed in light of changes in adhesive properties in the Sey brain that might control segregation, assembly and cell migration of progenitors of specific forebrain regions.

Abbreviations: AA, Amygdala; AC, Anterior commissure; ACX, Archicortex; AD, Hypothalamic nucleus, anterodorsal; AEP, Anterior entopeduncular area; AH, Anterior hypothalamus; CGE, Caudal ganglionic eminence; ChPL, Chorioid plexus; CP, Caudate-putamen; DB, Diagonal band; DT, Dorsal thalamus; DM, Hypothalamic nucleus, dorsomedial; EMT, Eminentia thalami; EP, Entopeduncular nucleus; EML, External medullary lamina; ET, Epithalamus; fF, Field of Forrel; GP, Globus pallidus; $\mathrm{Hi}$, Hippocampal primordium; HCC, Hypothalamic cell cord; HT, Hypothalamus; IN, Infundibulum; Is, Isthmus of mesencephalon; LGE, Lateral ganglionic eminence; LV, Lateral ventricle; MA, Mammillary area, basal region of P4;

M, Mesencephalon; Mb, Midbrain; MGE, Medial ganglionic eminence; NCX, Neocortex; OB, Olfactory bulb; OS, Optic stalk; P1-P6, Prosomeres 1-6 ; PA, pa, Paraventricular nucleus ; PC (in Fig. 1), Piriform cortex; PEP, Posterior entopeduncular area; POA, Anterior preoptic area; POP, Posterior preoptic area; PC, Posterior commissure; PT, Pretectum; r 1-r 7, Rhombomeres 17; RCH, Retrochiasmatic area; RP, Rathke's pouch; RT, Reticular thalamic nucleus; SE, Septum; SCH, Suprachiasmatic area; SPV, Supraoptic/paraventricular area; STM, Stria medullaris; $\mathrm{Tg}$, Tegmentum of the mesencephalon; TU, Tuberal hypothalamus, basal zone of P5; VLG, Ventral lateral geniculate body;

VM, Hypothalamic nucleus, ventromedial; VT, Ventral thalamus; ZI, Zona incerta; ZL, Zona limitans intrathalamica; 3V, Third ventricle

Key words: Pax6, Dlxl, Small eye, forebrain, patterning, mouse, brain development

\section{INTRODUCTION}

Recent evidence suggests that the patterning of the embryonic forebrain in vertebrates is specified by distinct sets of regulatory molecules, which are activated during a specific time window in development. Many of these genes are members of homeodomain encoding gene families like $N k x, D l x, E m x$, Otx, Pax, POU (reviewed in Alvarez-Bonaldo et al., 1995; Boncinelli, 1994; Chalepakis et al., 1993; Rosenfeld, 1991; Rubenstein and Puelles, 1994) or are winged-helix genes like $B F-1$ and BF-2 (Hatini et al., 1994) and secreted factors from the Wnt gene family (reviewed in McMahon, 1992; Nusse and Varmus, 1992). Their specific expression within the neuroepithelium of the developing forebrain is restricted at putative neuromeric boundaries (Boncinelli, 1994; Bulfone et al., 1993; Figdor and Stern, 1993; Price et al., 1992; Roelink and Nusse, 1991; Stoykova and Gruss, 1994). This lent support to the idea that similarly to the organisation of the hindbrain (reviewed in Guthrie, 1996; Krumlauf, 1994; Lumsden, 1990), the forebrain neuroepithelium may be compartmentalised into transverse domains (neuromeres) with distinct molecular properties. Two comparable models for the possible neuromeric organisation of the chick diencephalon (Figdor and Stern, 1993) and for the murine forebrain (Bulfone et al., 1993; Puelles and Rubenstein, 1993) have been recently proposed, which provide a framework for pattern analysis. Different aspects of the prosomeric model have been supported in recent gene expression (Shimamura et al., 1995; Timsit et al., 1995; Tole and 
Patterson, 1995) and axonal tract tracing studies (Mastick and Easter, 1996).

Changing the expression of the brain patterning genes is expected to produce alterations in the developmental program and should result in dysgenesis of the respective brain structures. The Pax genes encode evolutionary conserved transcriptional regulators (Bopp et al., 1986; Dressler et al., 1988; Wallin et al., 1993; Walther et al., 1991). Most of them are expressed in the developing and mature nervous system (Stoykova and Gruss, 1994), implicating functions in patterning, differentiation and maintenance of specific sets of brain cells (reviewed in Chalepakis et al., 1993; Mansouri et al., 1994). The remarkable feature of Pax6 is that it is the earliest expressed gene of the Pax family that is almost exclusively confined to the developing CNS, including the forebrain and the eye (Walther and Gruss, 1991). The Pax6 protein is strongly conserved throughout evolution. Genetic lesions in Pax6 cause a broad spectrum of ocular abnormalities in the Small eye (Sey) mouse (Hill et al., 1991; Hogan et al., 1986, 1988; Schmahl et al., 1993) and rat (Fujiwara et al., 1994; Matsuo et al., 1993), in humans with aniridia syndrome (Glaser et al., 1994; Jordan et al., 1992; Ton et al., 1991) or with Peter's anomaly (Hanson et al., 1994) and in the Drosophila eyelessmutant (Quiring et al., 1994). The misexpression of eyeless and of murine Pax6 in the imaginal disc primordia induces the formation of ectopic eye structures in Drosophila, demonstrating the functional conservation of Pax6 during evolution and its essential role in the eye formation (Halder et al., 1995). Finally, in the nematode C. elegans Pax-6 is involved in patterning of the head region (Chisholm and Horvitz, 1995; Zhang and Emmons, 1995).

We have previously reported a particularly strong and developmentally early restricted expression domain of Pax6 in the anlage of telencephalic cortex and in the ventral thalamus (Stoykova and Gruss, 1994; Walther and Gruss, 1991). Cortex malformations have been observed in homozygotes of the Small eye allele Seyneu (Schmahl et al., 1993) and, in one case, of human homozygous aniridia (Glaser et al., 1994). Intriguingly, no severe pathology in the specification of particular diencephalic structures has been reported, except for a dilatation of the third ventricle in Seyneu (Schmahl et al., 1993) as well as a dysgenesis of the anterior diencephalon in human homozygous aniridia (Glaser et al., 1994).

To define the developmental role of Pax6 in the formation of the forebrain, we investigated the expression pattern of Pax6 and other genes in conjunction with the malformations of the Sey brain. Here we show that early developmental commitment of the transcription factors Pax6 and Dlxl to distinct neuromeric fields in the wild-type brain correlates with the specification of particular diencephalic nuclei. Loss of Pax6 function, as seen in Sey, causes a failure of the establishment of several expression boundaries that specify longitudinal domains within the developing forebrain. Consequently, the development of the hypothalamo-telencephalic transition zone and of distinct forebrain structures is distorted.

\section{MATERIALS AND METHODS}

\section{Animals}

The allele of the Small eye mouse used in this work is Sey, a sponta- neous mutation originally described by Roberts (1967) and Hogan et al. (1986), kept under the background C57BL/6J×DBA/2J. The day of the vaginal plug was considered as stage E0.5 dpc. Homozygous (Sey/Sey) and wild-type (WT) littermate embryos with developmentally matched stages were obtained from crosses of heterozygous Small eye parents, selected on the basis of the eye size. Embryos were removed to ice-cold phosphate-buffered saline (PBS) and fixed in $4 \%$ paraformaldehyde in PBS at $4^{\circ} \mathrm{C}$. The next day they were dehydrated through ethanol/saline solutions and after xylene treatment, specimens were embedded in Paraplast (Monoject Scientific).

\section{In situ hybridisation}

Sectioning, in situ hybridisation, washing and emulsion autoradiography were performed as previously described (Stoykova and Gruss, 1994). ${ }^{35}$ S-labelled RNA probes were synthesised in the presence of two radioactive nucleotides according to the supplier's directions (Promega) from corresponding linearized plasmid templates as described for: Dlx 1 in Price et al. (1991); Emx2 in Simeone (1992b); Mash1 in Guillemot and Joyner (1993); Otx2 in Simeone et al. (1993); Pax6 in Walther and Gruss (1991); Proxl in Oliver et al. (1993), Wnt3 and Wnt3a in Salinas and Nusse (1992). Two independent in situ hybridisation analyses were performed for each stage on serial sagittal, frontal and transverse sections from wild-type and Sey/Sey littermate embryos. The terminology for the prosomeric subdivision of the forebrain is according to Bulfone et al. (1993) and Puelles and Rubenstein (1993). For the regional identification of the developing brain, we used the rat brain atlas of Paxinos et al. (1991) and Altman and Bayer (1995).

\section{RESULTS}

\section{Complementary expression domains of Pax6 and DIx1 define boundaries in the E12.5 dpc forebrain}

At stage E12.5 dpc previous studies suggested the existence of overlapping and complementary expression domains for Pax6 and Dlxl in the diencephalon and in the secondary prosencephalon (Bulfone et al., 1993; Porteus et al., 1991; Price et al., 1991; Puelles and Rubenstein, 1993; Walther and Gruss, 1991). To obtain direct experimental evidence for the specific regionalization of the forebrain by Pax 6 and $D l x l$, we initially performed in situ hybridisation on serial sagittal, cross and frontal sections from wild-type embryos, using Pax6, Dlxl and several other forebrain markers as probes. The results are presented in Figs 1 and 2 and are summarized in Fig. 7. For terminology, we refer to the prosomeric model of Bulfone et al. (1993) and Puelles and Rubenstein (1993).

Consistent with the reported data (Bulfone et al., 1993; Price et al., 1991), Dlxl expression is detected in two large domains. The telencephalic domain includes the regions of: septum (SE), diagonal band region (DB), lateral and medial ganglionic eminence (LGE, MGE), anterior entopeduncular area (AEP), anterior preoptic area (POA). Within its thalamo-hypothalamic expression domain Dlxl colocalizes with Pax6 within the regions of the ventral thalamus (prosomere 3 ), in the posterior entopeduncular area (PEP, prosomere 4) and specifically delineates the regions of the hypothalamic cell cord (HCC, prosomere 5) and the suprachiasmatic area $(\mathrm{SCH}$, prosomere 6). Between the telencephalic and thalamo-hypothalamic expression domains of $D l x l$, a forebrain region designated as 'optoeminential zone' does not show any $D l x l$ hybridisation signal (Bulfone et al., 1993). It consists of the caudal ganglionic eminence (CGE), the eminentia thalami (EMT), the supraoptic paraventricular area (SPV), the anterior hypothala- 
mus (AH) and the posterior preoptic area (POP). As shown in Fig. 1 (also Fig. 7), all these domains strongly hybridise with the Pax6 probe. These results are direct evidence that the optoeminential zone is a Pax6-positive domain within the secondary prosencephalon that abuts the rostral and the caudal Dlxl expression regions, as suggested in the prosomeric model. It should be noted, however, that we detected a small Dlxlpositive subdomain within the SPV (Figs 1F, 2N). The complementary expression of Pax6 and Dlxl within the secondary prosencephalon defines boundaries at the levels of: neocortex/lateral ganglionic eminence (NCX/LGE), posterior preoptic area/anterior preoptic area (POP/POA) and posterior preoptic area/suprachiasmatic area (POP/SCH, Figs 1A,B, 2A,B); supraoptic paraventricular area/hypothalamic cell cord (SPV/HCC, Fig. 1E,F). When related to the topological map with an extended longitudinal axis of the brain (Puelles and Rubenstein, 1993), they appear as longitudinal borders outlining different strata in the forebrain.

In addition, Pax6 and Dlxl expression domains outline a well-defined transverse neuromeric border, the zona limitans intrathalamica (ZL) that separates the dorsal (prosomere 2) from the ventral thalamus (prosomere 3; Fig. 2M,N) and also another transverse expression border between the EMT and AEP (arrows in Fig. 1E,F) which corresponds to a boundary between prosomere 4 and prosomere 5 as proposed in the prosomeric model. In the ventral thalamus, we found that Pax6 and $O t x 2$ but not the $D l x 1$ probe hybridises within the dorsalmost region (Fig. 2G-I). Interestingly, at this early stage, neighbouring domains within prosomere 4 (presumably representing the differentiating endopeduncular nucleus (EP) and a region in the lateral hypothalamus) are specifically patterned by Pax6 or by Dlxl, respectively (arrows in Fig. 1I,J).

\section{Failure of the formation of expression boundaries and erroreous growth of forebrain primordia in the Sey/Sey brain at stage E12.5 dpc}

Next, we analysed the homozygous Small eye brain (Sey/Sey) and compared the patterning of the wild-type and the mutant brains at corresponding levels by in situ RNA hybridisation analysis. In accordance with the reported switch off of the Otx2 expression at E11.75 dpc in the cortical domains (Simeone et al., 1993), the hybridisation signal is absent in the wild-type and also in the Sey/Sey cortex (Fig. 2C,I,F,L), indicating that there is no general unspecific delay of the differentiation program in the mutant brain. Different regions of the cortical primordia were adequately labelled by genes that are expressed in the future neocortical $(E m x 2$, Otx1, Otx2) or archicortical areas (Wnt3a, data not shown). Nevertheless, the cortical primordia are severely underdeveloped and the archicortex anlage fails to invaginate along the medial wall of the telencephalic vesicle.

The developing diencephalon of the mutant brain shows a surprising phenotype. The primordia of the EMT (prosomere 4) and the very rostrodorsal part of the ventral thalamus (prosomere 3), which are specifically labelled by Pax6 and Otx 2 but not by Dlx 1 (Fig. 2G-L), grow in the wrong direction. Instead of growing rostroventrally in order to fuse with the MGE and AH at the basis of the forebrain, these regions in the Sey/Sey forebrain grow laterally (compare WT/SEY in Fig. 1, levels 2/2"; 3/3"; Fig. 2, levels 2, 3). This results in an abnormally large third ventricle, making wide connection with the left and the right forebrain ventricles. The partition of the ventral thalamus is seen only at its dorsalmost regions, while the caudal diencephalon (where Pax6, Dlx1, Otx2 extensively overlap) looks relatively normal except for an enlargement of the third ventricle (Fig. 1, level 4/4"; Fig. 2. level 2, 4). In addition, the circular boundary zone at the level of the ZL which is normally strongly labelled by Otx2 (Simeone et al., 1993) is not detectable in the Sey/Sey diencephalon (arrowhead in Fig. 2I/L,P).

Within the hypothalamus, the ventricular zone of the region SPV (prosomere 4) seems to be correctly labelled by Pax6 (arrow in Fig. 1G). However, in absence of functional Pax6 protein, the compartmentalisation within the hypothalamus is distorted, the expression of Dlxl becomes widely dispersed and extends beyond the normal sharp boundaries at the levels of POA/POP, SCH/POP (Fig. 1B/D) or HCC/AH (Fig. 1F/H). Neither the EP nucleus (presumably a derivative of PEP, prosomere 4; Pax6 positive) nor the neighbouring structure in the lateral hypothalamus ( $D l x l$ positive) could be observed in the mutant brain (compare Fig. 1I-L). As shown in Fig. 1D,H and under higher magnification in Fig. 6A,B the expression boundary for $D l x l$ at the level LGE/NCX is also distorted.

Taken together, these results show two main disturbances in the homozygous Small eye brain at stage E12.5 dpc: (a) severe growth disturbances of forebrain regions that are specifically patterned by Pax6, which affect the formation of the transition zone between the telencephalic vesicle and hypothalamus (prosomere 3, prosomere 4); (b) failure of the restrictive capacity of several expression boundaries for $D l x l$ within the secondary prosencephalon.

\section{Spatially restricted expression of Pax6 and $D / x 1$ in the differentiating ventral thalamus and hypothalamus at stage E14.5 dpc}

In the wild-type forebrain, the paraventricular nucleus (PA) and a stream of cells extending all along the external medullary lamina that presumably contribute to the formation of a part of the ventral lateral geniculate body (VLG) hybridise strongly with the Pax6 probe at E14.5 dpc (arrows in Fig. 3A, empty arrowhead in 3C). Only a small subpopulation of cells in these two regions express Dlxl (arrows in Fig. 3B), which in contrast is detected in the entire region of the differentiating VLG (empty arrowhead in Fig. 3D) and the hypothalamic anterodorsal nuclei (ad, AD Fig. 3B,D). The comparison of the expression of Pax6 and Dlxl within the ventral thalamus reveals a complementary pattern. Within the central part of the ventral thalamus, Pax6 is excluded from the Dlxl-positive domain, which presumably represents the differentiating zona incerta (ZI) and the reticular thalamic nucleus (RT; arrow in Fig. 3C,D). Only Pax6 is strongly expressed in the prospective entopeduncular nucleus (EP, arrowhead in Figs 3C,D, 4B). In more caudal levels, an extensive overlap is detected for Pax6 and Dlxl. The results show that, at stage E14.5, the expression of Pax6 is mainly confined to the paraventricular and entopenduncular nuclei and a subregion of the ventral lateral geniculate body, while Dlxl expression is restricted to the prospective zona incerta, reticular nucleus, entire ventral lateral geniculate body and hypothalamic anterodorsal nucleus.

\section{Disturbed development of diencephalic structures in the Sey/Sey brain at stage E14.5 dpc}

In the mutant brain, the thalamus appears to be of an earlier 


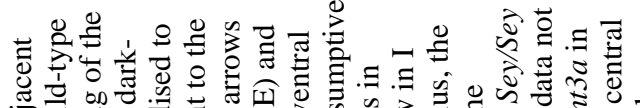

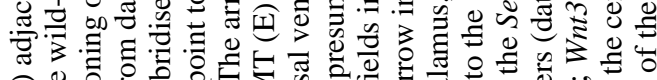

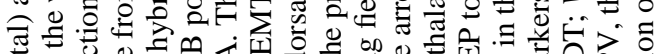

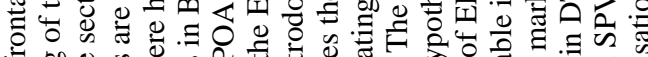
>.

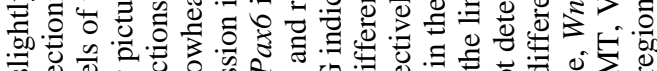

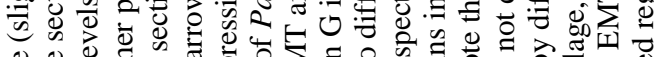

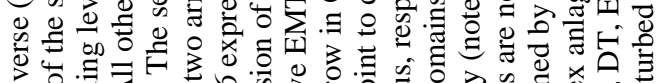

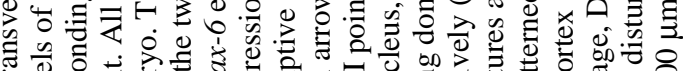

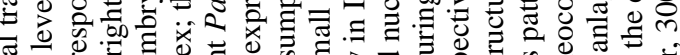

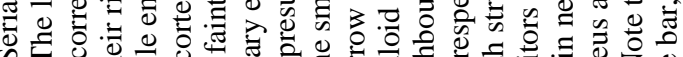
ن.

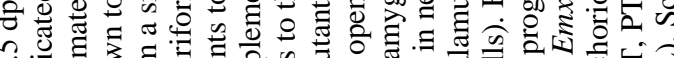

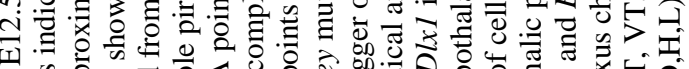

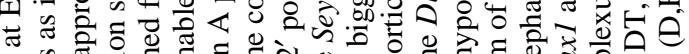

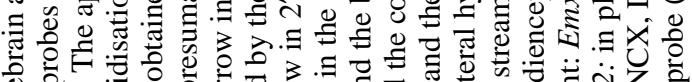

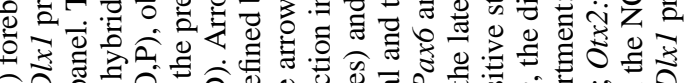
a

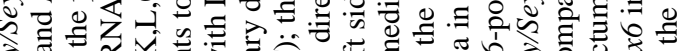

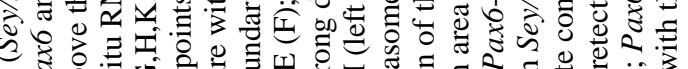

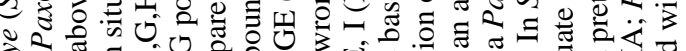

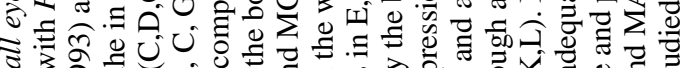
ह

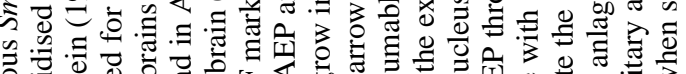

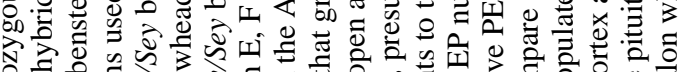

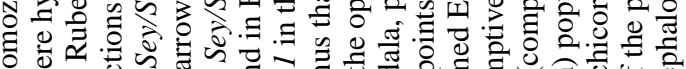

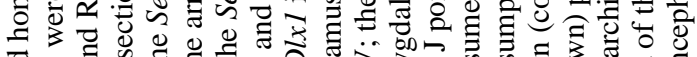

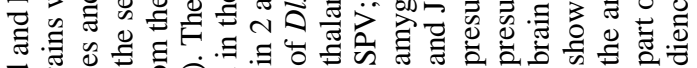

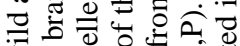

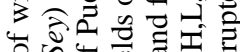
ठै o d क

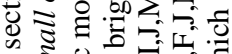

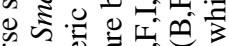

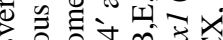
bo

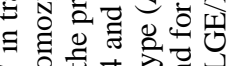

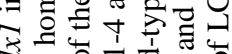

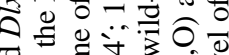

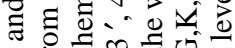
$0=0$ हैं पै

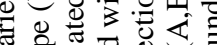

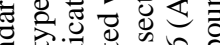

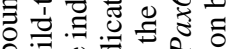

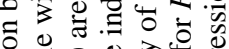

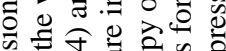
o

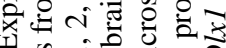
I

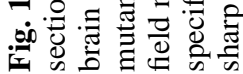
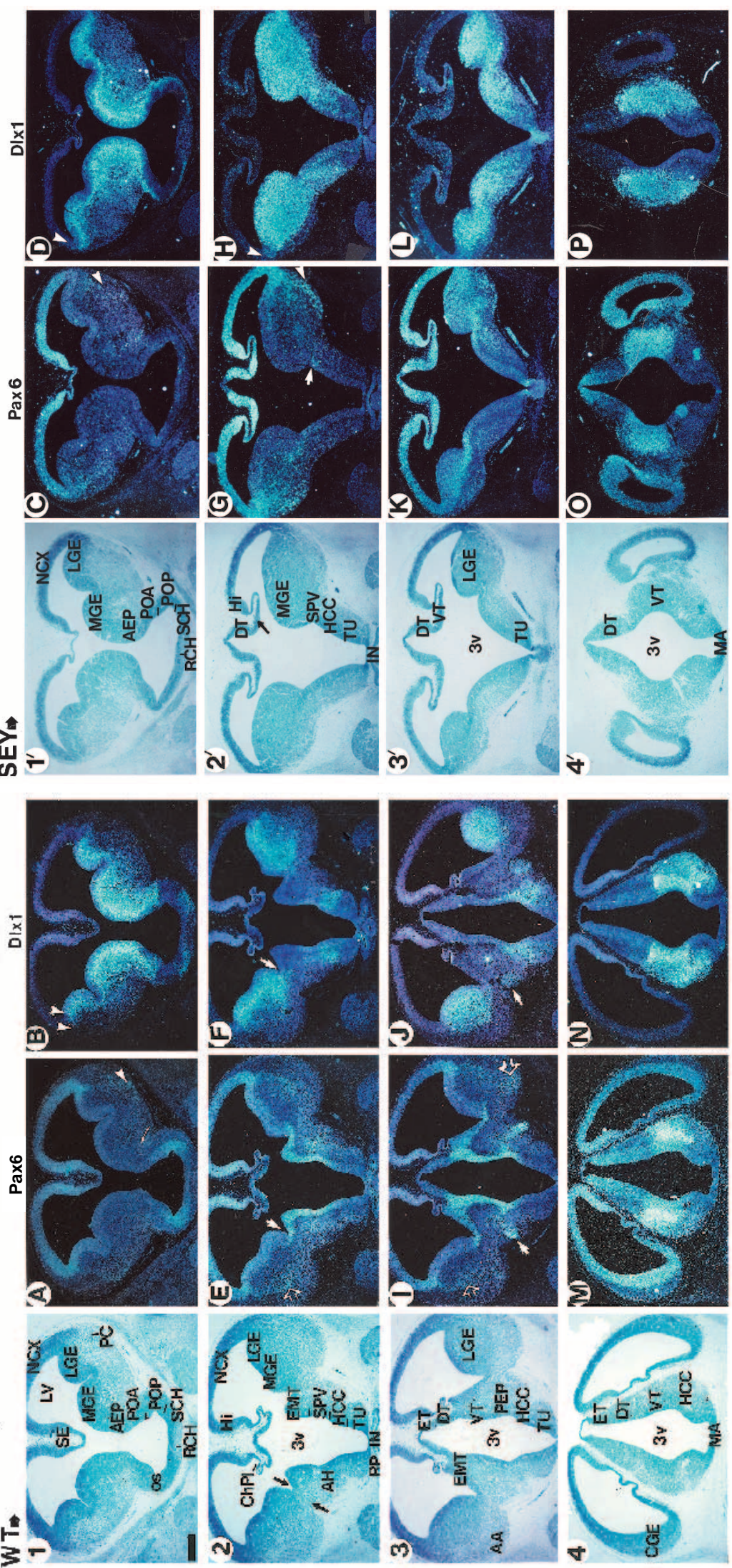
Forebrain patterning and the function of Pax6 3457
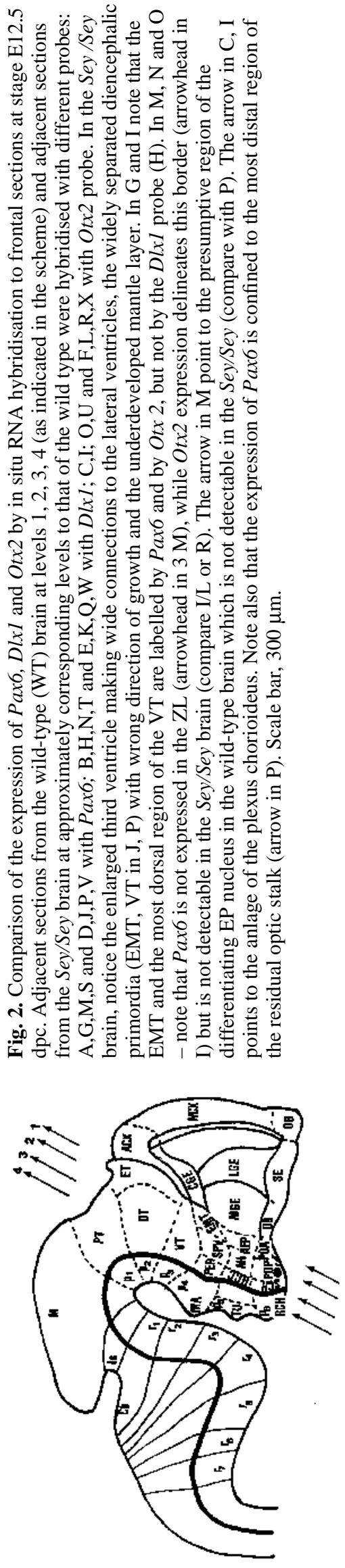
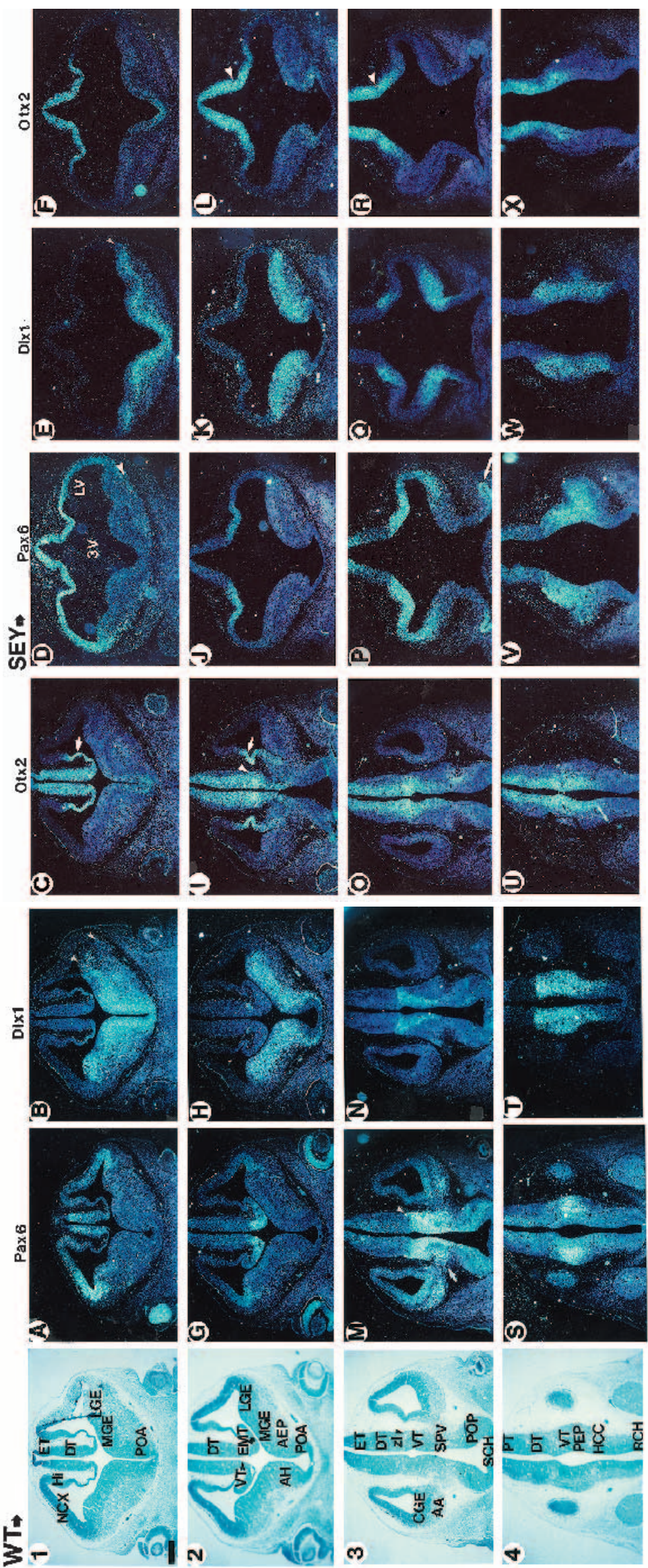

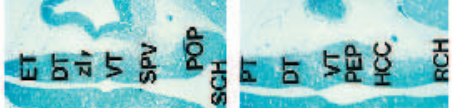
픙혀 

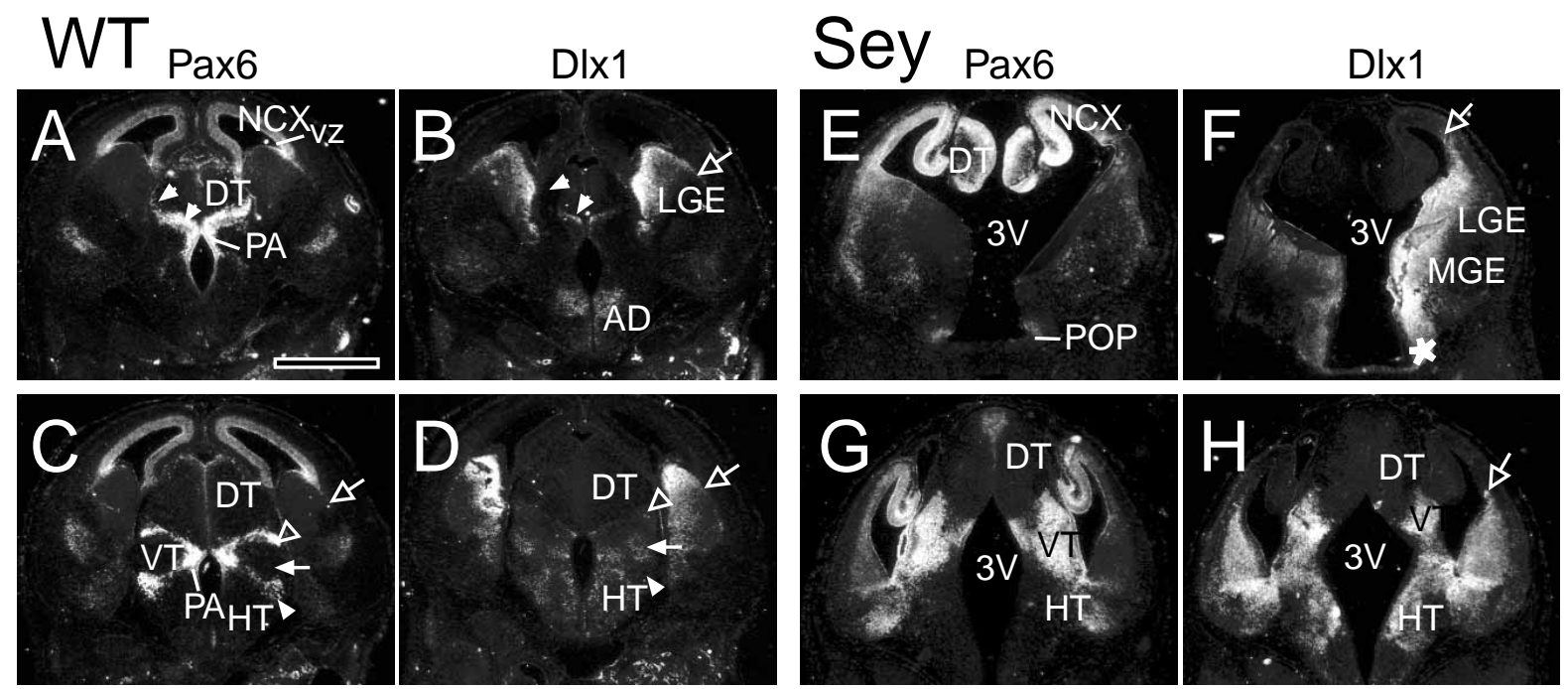

Fig. 3. Complementary expression of Pax6, and Dlxl in the wild-type forebrain and pattern disturbances in the Sey/Sey forebrain at stage E14.5 dpc. (A-D) Adjacent transverse (slightly frontal) sections through a wild-type forebrain with A, B at more rostroventral and C, D at more dorsocaudal plane where hybridised with $\operatorname{Pax} 6(\mathrm{~A}, \mathrm{C})$ or $\operatorname{Dlxl}(\mathrm{B}, \mathrm{D})$. The big open arrow in B points to the sharp limit of the Dlxl expression at the level of LGE/NCX. The open arrowheads in C and D point to distinct parts of the VLG, expressing Pax6 or Dlxl, respectively. The filled arrows in $\mathrm{C}$ and $\mathrm{D}$ show the presumptive region of the differentiating zona incerta and the reticular nucleus, that are specifically labelled by the Dlxl probe; the filled arrowheads point to the prospective EP, labelled by the Pax6 probe, but not by Dlxl. (E-H) Two levels of transverse sections through the Sey/Sey mutant forebrain. (G,H) Sections approximately comparable to the level C,D in the wild-type brain, while (E,F) more ventral planes. The empty arrows in F, H point to the expression of Dlxl in the cortical areas extending far beyond the LGE/NCX border. Note the extension of Dlxl expression into POP, which is normally patterned only by Pax6 (compare E/F; star). Note the enlargement of the third ventricle, the partition of the dorsal thalamus that has an internal germinative layer still actively expressing Pax6 (compare with A, B) and the failure of the compartmentalisation of the expression domains for Pax6 and Dlxl within the ventral thalamus. Scale bar, $1 \mathrm{~mm}$.

developmental stage; left and right primordia are smaller and widely separated from each other, the internal germinative layer (IGL) of dorsal thalamus is still strongly expressing Pax6 (Fig. 3E). Previously, we reported that, around E13.5 dpc, the main expression domain of the homeobox gene Proxl (a vertebrate homologue of the Drosophila prospero gene) is translocated from ventral to the dorsal thalamus, following the developmental dynamic of the differentiation processes (Oliver et al., 1993). At E14.5 dpc, we found an abundant expression of Proxl in the mantle zone of the dorsal thalamus of the Sey/Sey brain (data not shown), showing that despite the inadequate growth there is no severe general delay in the differentiation program of the diencephalon in the mutant brain. As illustrated in sagittal (Fig. 4E-H) and transverse sections (Figs 3E,F, 6E$\mathrm{H})$, there is a paucity of tissue in the mutant brain, encompassing regions of the optoeminential zone. Therefore, the comparison of the patterning between the wild and the mutant brain at corresponding levels is possible only in more caudal sections as shown in Fig. 3C,D/G,H. Consistent with the abnormal bifurcation and lateral displacement of the rostrodorsal regions of ventral thalamus already noticed at E12.5 dpc, we found that Pax6 and Dlxl extensively label only the lateralmost diencephalic regions (Fig. 4E-H). Nevertheless, none of the structures that are specifically patterned by Pax6 or Dlxl could be distinguished either in lateral or transverse sections in the mutant brain. Taken together, these results show that the regions of the optoeminential zones, which are specifically patterned by $\operatorname{Pax} 6$ at E12.5, are severely affected in the $\mathrm{Sey} / \mathrm{Sey}$ brain in later stages. This results in a paucity of tissue within the hypothalamus. Furthermore, the specification of distinct nuclear structures within ventral thalamus through the restricted expression of Pax6 or Dlxl is abolished.

It is of special interest to note that, at E14.5 dpc, the abnormal expression of Dlxl in the cortical areas in the Sey/Sey forebrain has further expanded (Figs 4F,H, 6A-D).

\section{Differential patterning of forebrain structures by Pax6 and DIx1 at E18.5 dpc}

To adequately correlate the observed restricted expression of Pax6 and Dlxl in the developing forebrain, we followed the expression of these genes also at prenatal stage. On frontal sections through the rostral diencephalon, the paraventricular nucleus and the entopenduncular nuclei hybridise strongly with the Pax6 probe (Fig. 5A,C,E). Complementary to Pax6, a diffuse Dlxl signal is observed in the region of the rostral zona incerta and the reticular nucleus (Fig. 5B,D), which is detected also in few cells in the paraventricular nucleus. Similarly to the observations at E14.5 dpc, Pax6 is expressed in a stream of cells which delineates the $D l x l$ expression caudally in the ventral thalamus and ends in a subregion of the VLG (two arrowheads in Fig. 5A,C). The Dlxl (Fig. 5D,F) and Otx2 transcripts (data not shown) are more abundant in lateral areas including the RT and the entire VLG. Different patterning is seen in more caudal levels. In general, the expression domains of both genes overlap within the PA and caudal ZI, except for the VLG, which hybridises exclusively with the Dlxl probe (also with Otx2, not shown). The Dlxl signal is fainter and more widely distributed within the inter- 

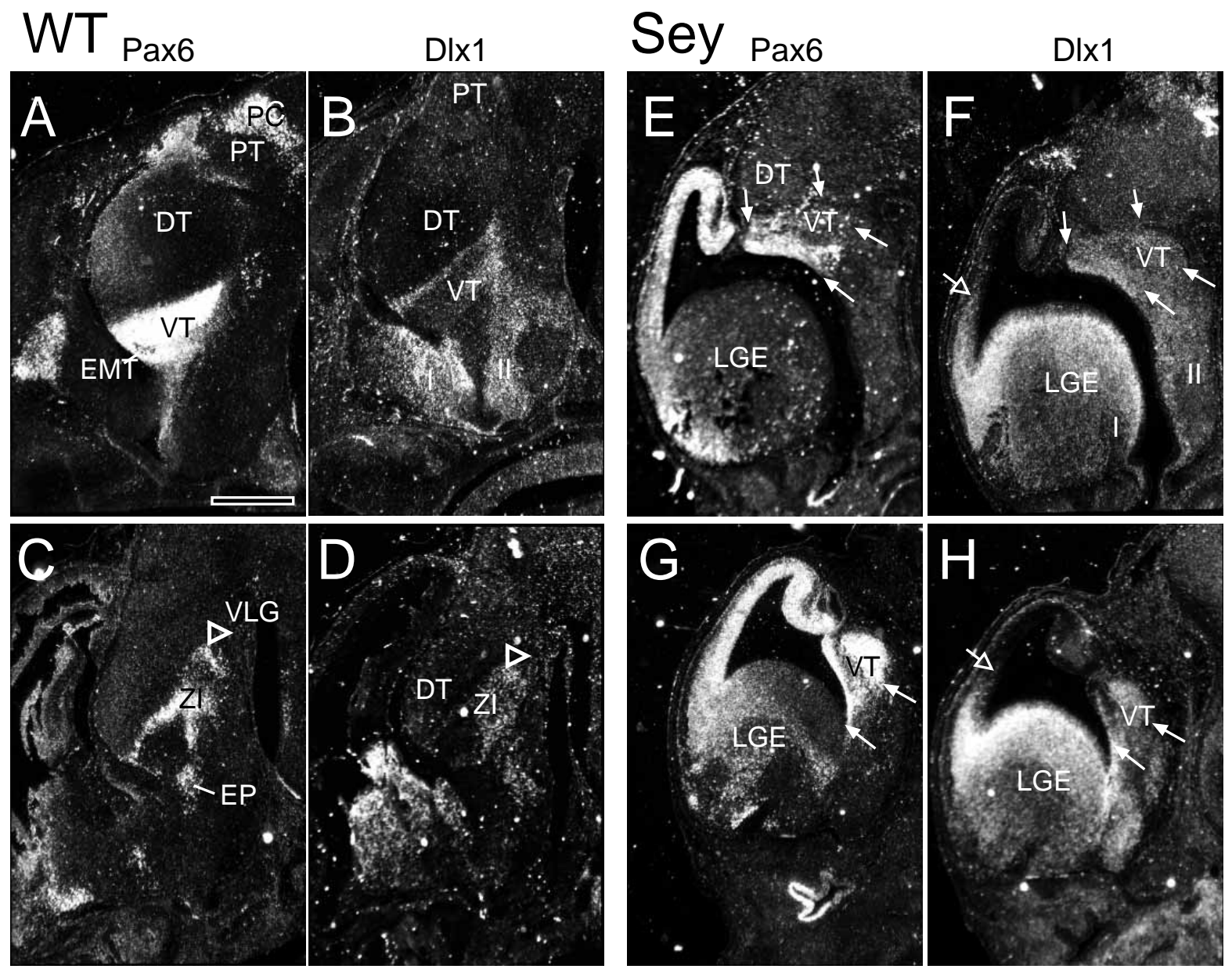

Fig. 4. (A-D) Expression of Pax6 and Dlxl on parasagittal sections of wild-type brain at stage E14.5 dpc. (A,B) Sections at more medial levels than C, D. Within the hypothalamus (HT), note that the opoeminential zone is a Pax6-positive region intercallated between the telencephalic (I) and thalamohypothalamic expression domain (II) of Dlxl. The two open arrowheads in C and D point to distinct regions of the presumptive VLG labelled either by Pax6 or Dlxl. The Pax6 and Dlxl expression domains overlap in the caudal region of the ZI. (E-H) Morphogenetic and pattern disturbances in Sey forebrain at stage E14.5. The sections of the mutant brain at E, F and G, H are at approximately corresponding levels to the sections of the wild-type brain at A, B and C, D, respectively. The empty arrows indicate the extension of the Dlxl expression domain into the telencephalic cortex. The region of the laterally displaced ventral thalamus is outlined by arrows. None of the structures that specifically express Pax6 or Dlxl can be distinguished. (F,H) Note the paucity of tissue corresponding to the region of the Pax6-positive optoeminential zone. Scale bar, $1 \mathrm{~mm}$.

mediate hypothalamus and the field of Forrel (fF), while the expression of Pax6 is mostly confined to the dorsocaudal parts of the ZI. More caudally, the Pax6 probe labels the region of the suprafasicular nucleus and the transcripts of both genes are detected in the dorsal and the ventral regions of the ZI (data not shown). In all planes, Dlxl hybridises within the region of the anterodorsal and the mediodorsal hypothalamic nucleus (Fig. 5B,D,F).

Taken together these results show that, in the diencephalon, the expression pattern of the two genes changes little from stage E14.5 dpc to E18.5 dpc, which allowed us provisionally to assign the fate of differently patterned prosomeric fields to distinct diencephalic structures (see Discussion).

At the prenatal stage, Pax6 transcripts are still detected within the dorsolateral ventricular zone and subventricular zone (Fig. 5A), while having background levels in these zones in the basal rostral telencephalon. On the contrary and as noticed in earlier stages, the Dlxl transcripts are abundant in the striatal and in the pallidal subventricular zone thus establishing a sharp boundary with the Pax6-positive region at the level of the caudate-putamen (Fig. 5B,D). At this stage, a faint (slightly above background level) signal is detected for Dlxl in the ventricular zone and subventricular zone of the dorsal telencephalon. Several palleocortical areas including the differentiating entopiriform nucleus, piriform cortex and olfactory tubercles show specific hybridisation with the Pax6 probe. Interestingly, the two markers label different differentiating nuclei of the amygdala along the rostrocaudal axis (Fig. 7). Rostrally, the presumptive cortical, the basolateral and the medial nucleus of the amygdala are outlined mainly by the Pax6 hybridisation signal whereas, in the more caudal planes, the central and the medial amygdaloid nuclei are Dlxl positive.

\section{Dysgenesis of the diencephalon of the prenatal Sey/Sey brain}

Consistent with the data of Schmahl et al. (1993), in Sey/Sey 

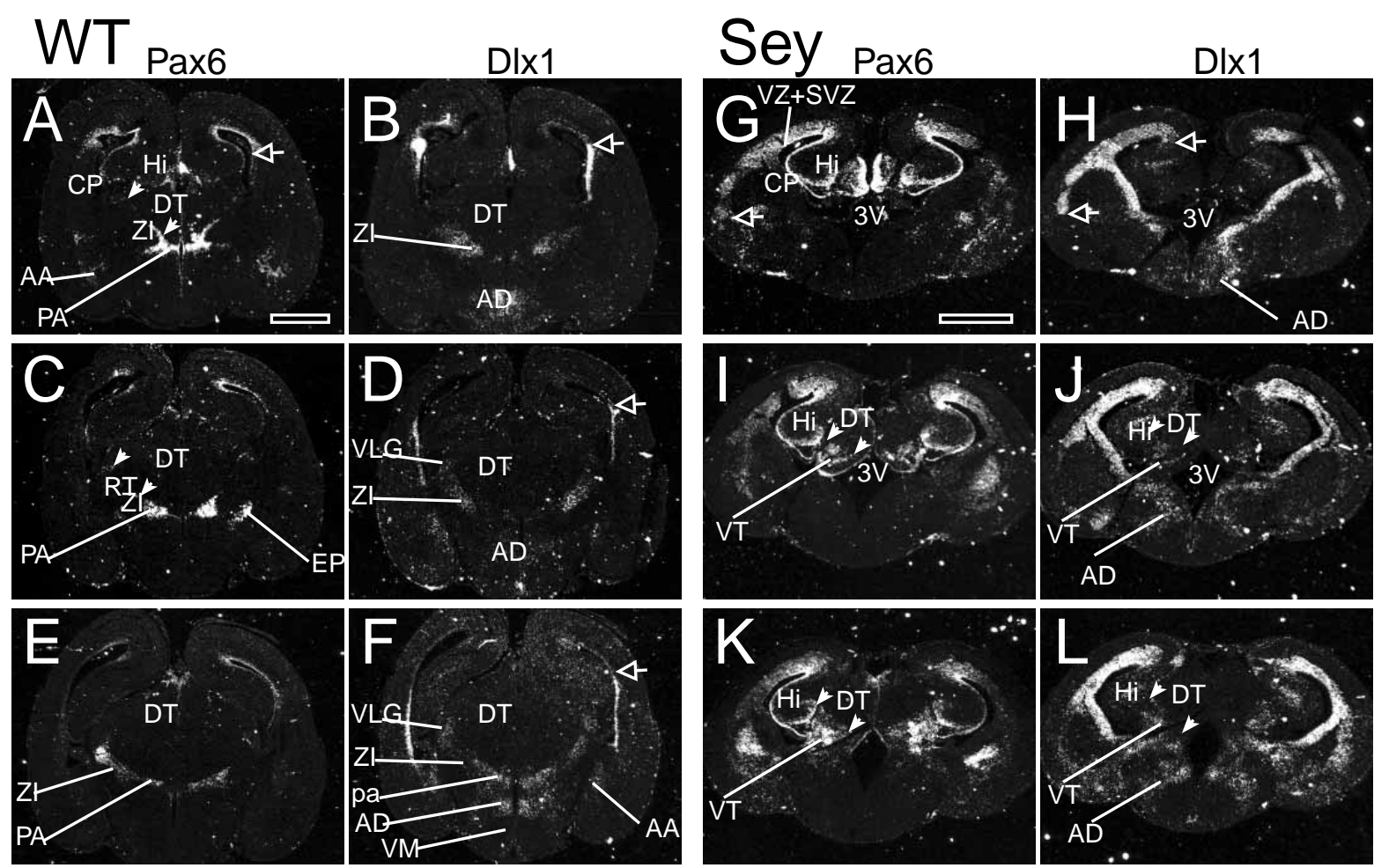

Fig. 5. Pattern disturbances in prenatal Sey/Sey brain. (A-F) Serial adjacent coronal sections from the wild-type brain with A, B at most rostral and E, F at more caudal levels of sectioning. Note the mostly complementary expression in the rostral diencephalon of Pax6 (PA, EP, subset of cells of VLG) and Dlxl (in the rostral ZI, VLG). The arrows in A, C point to the Pax6-positive stream of cells under the EML, presumably contributing to the formation of a part of the VLG; the empty arrow in B, D and F point to the sharp expression limit for Dlxl in the ventricular zone and SVZ of the forebrain. Note the extensive overlapping of the expression domains of the two markers within the PA, ZI and RT in more caudal planes (E,F). (G-L) Serial adjacent coronal sections through the diencephalon of the homozygous mutant forebrain with G, H at the most rostral and K, L at most caudal levels. Note the extensive overgrowth of the VZ and SVZ of the frontoparietal cortex and the nests of cells that express strongly both markers. These streams (open arrows in G) probably represent the lateral migratory stream of progenitors of VZ (giving rise to the piriform cortex; Altman and Bayer, 1995) and/or claustrum. The arrowheads in I-L point to the border between DT and VT. Note the severe morphogenetic disturbances in the rostral diencephalon and the incorrect location of PA, rostral ZI and RT. The morphology in the caudal diencephalon is less severely affected; note the enlarged third ventricle making wide connection with the lateral ventricles. Scale bar, $1 \mathrm{~mm}$.

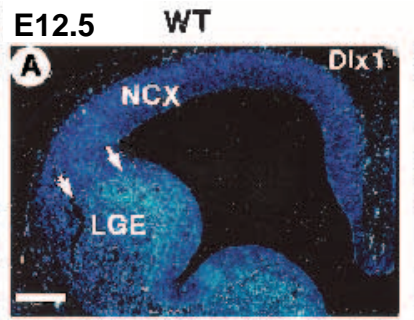

\section{E14.5}

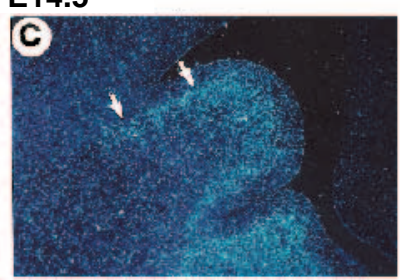

SEY
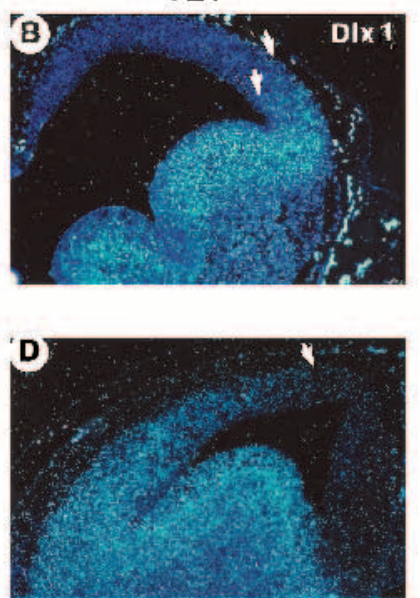

$\mathrm{E} 14.5 \mathrm{WT} \rightarrow$
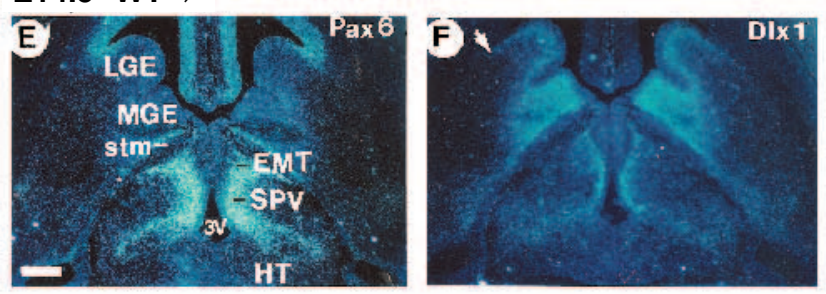

$S E Y \rightarrow$
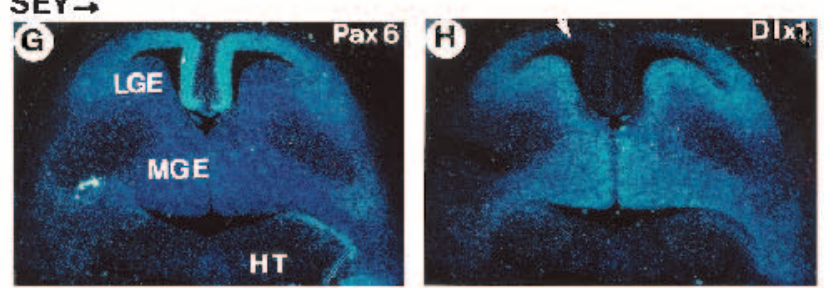

Fig. 6. Details of the pattern disturbances in the Sey/Sey brain at different developmental stages. (A-D) The expression limit for Dlx1 at stage E12.5 dpc (A,B) and at stage E14.5 (E,F) on transverse sections at the level of the NCX/LGE in the wild-type brain (A,C) and in the Sey/Sey brain (B,D). The arrows in A, C point to the normal sharp expression boundary for Dlx1 at E12.5 dpc (A) and at E14.5 dpc (C). In the Sey/Sey, the Dlxl signal is detected in the cortical area already at stage E12.5 (arrows in B) and is furthermore extended at stage E14.5 dpc (arrow in D). Scale bar, $150 \mu \mathrm{m}$. (E-H) Adjacent transverse sections from the wild-type (E,G) and from the Sey/Sey brain (F,H) of E14.5 dpc embryos at corresponding levels hybridised with the $\operatorname{Pax} 6(\mathrm{E}, \mathrm{G})$ and with the $\operatorname{Dlxl}(\mathrm{F}, \mathrm{H})$ probes. The figure illustrates the medial extension of the MGE at a most rostrobasal level substituting the paucity of diencephalic structures (presumably at this level the EMT, SPV) from their normal locations. Scale bar, $300 \mu \mathrm{m}$. 
Fig. 7. Summary diagrams of the patterning by Pax6 and $D l x l$ in the wild-type and the Sey/Sey forebrain during embryogenesis. (A) Mapping of the expression pattern of Pax6 (red) and Dlxl (yellow) at stage E12.5 dpc on the prosomeric model of Puelles and Rubenstein (1993). According to this model the forebrain is divided into six transverse neuromeric regions (prosomeres, P) that relate to the restricted expression domains of numerous regulatory genes. $\mathrm{P} 1, \mathrm{P} 2$, P3 form the diencephalon and correspond to neuromeric regions of pretectum (PT), dorsal thalamus (DT) and ventral thalamus (VT). P4, P5, P6 constitute the secondary prosencephalon assumpted to contain in their basal domains the hypothalamus while including the telencephalic cortex and optic vesicle into evaginations of their alar domains. Expression boundaries also define hypothetical longitudinal zones, parallel to the longitudinal axis of the neural tube that follow the ventral and the dorsal midlines as recently documented (Shimamura et al., 1995). As a result, the forebrain is subdivided into compartments each containing distinct molecular characteristics. In the schema, different symbols in the corresponding colour are used to indicate descendants of presumptive histogenetic fields within prosomere 3 and prosomere 4 , as suggested by the results of this study. Within prosomere 3 , the triangles and the squares represents the differently patterned descendants, presumably corresponding to the 'pars geniculata' and the 'pars inserta' of the suprapeduncular complex, while the hexagons indicate the descendats of a matrix that presumably corresponds to the 'pars ventralis thalami' (Keyser, 1972). The dots within the PEP of prosomere 4 represent the accumulation of Pax6-patterned descendents of this histogenetic fields within the presumable region of the EP nucleus that borders on a Dlx 1 patterned nuclear structure within the lateral hypothalamus. The yellow asterisks represent descendents of SPV labelled by Dlxl. The arrow that extends from PEP marks the presumptive pathway for migration of the EP nucleus. (B-E) Expression of Pax6 and Dlx 1 in the developing diencephalon and cortex at stage E12.5 dpc (B), E14.5 dpc (C) and at stage E18.5 dpc at rostral (D) and caudal (E) level. The corresponding symbols are used to indicate the presumptive origin of nuclei and structures from distinct histogenetic fields. The dashed line in $\mathrm{D}$ indicates that the presented information for the patterning of the cortex concerns a more rostral level than that for the diencephalon. (F-J) Developmental pattern disturbances in the Sey/Sey diencephalon at corresponding levels to that of the wild-type brain at stage E12.5 dpc (F), E14.5 dpc (G) and at stage E18.5 at rostral $(\mathrm{H})$ and caudal level (J). 


\section{A. Stoykova and others}

we observed a constant failure of proper cortical lamination, extensive overgrowth of the ventricular zone and subventricular zone of the frontoparietal cortex, cortical heterotopias, absence of anterior commissure and severe underdevelopment of the internal capsule, corpus callosum, posterior commissure and the basal ganglia (Fig. 5C-L). The sharp border between the expression domains of Pax 6 and Dlxl in the ventricular zone and subventricular zone of the frontoparietal cortex is lacking in the mutant forebrain. Here the entire ventricular zone and the subventricular zone of the telencephalon, including the striatal, the neocortical regions and also the superficially located islands of germinative cells (the cortical heterotopias) are strongly labelled by both markers (Fig. 5G-L). For Mash1 (a marker for relatively undifferentiated neuroblasts), similar hybridisation signals were detected in the telencephalic ventricular zone/subventricular zone in the wild-type and in the mutant brain. This suggests that the bulk of the constituents of the pathological subventricular zone in the cortex of the Sey/Sey mutant are presumably in a postmitotic state (reported also for the Sey ${ }^{\text {neu }}$ allele (Schmahl et al., 1993). However, the proliferation and differentiation states of the Sey cortical cells need a direct examination in a birthday study with markers for differentiated postmitotic neurons.

The observed early pattern and morphological abnormalities within the rostral diencephalon in the Sey/Sey forebrain are maintained through E18.5 dpc. At this developmental stage, the rostral zona incerta and the reticular nucleus (specifically patterned by $D l x l$, Fig. 5B,D) and the paraventricular nucleus (mainly patterned by Pax6; Fig. 5A,C,E) should have migrated to their final, rostralmost locations between the dorsal thalamus and the anterior hypothalamic area. In the Sey/Sey brain, these structures are absent from their normal position (Fig. 5G,H), the third and the lateral forebrain ventricles are connected through wide openings (instead of through the normal thin foramina Monro) and the left and the right sides of the diencephalon are not fused. Noteworthy, the entopeduncular nucleus (specifically labelled by $P$ ax6) is also not detectable at its normal location within the cerebral peduncle. Caudally, the regions of the paraventricular nucleus, zona incerta and ventral lateral geniculate body do not show their characteristic patterning for the applied markers (Fig. 5IL) and the third ventricle is still enlarged, due to the underdevelopment of the paraventricular nucleus and the caudal zona incerta (which normally extends medially to the lumen of the ventricle). The patterning of the anterodorsal nuclei by the $D l x l$ gene within the hypothalamus seems to be unaffected (Fig. 5H,J,L).

\section{DISCUSSION}

\section{Restricted expression of the transcriptional factors Pax6 and DIx1 and the patterning of the diencephalon}

Pax6 is expressed in restricted domains of the forebrain beginning at E8.5 dpc but also in the midgestation and in the mature brain (Stoykova and Gruss, 1994; Walther and Gruss, 1991). To evaluate the possible involvement of Pax6 in regional specification of the forebrain, we compared the morphology and the patterning of the developing forebrain in wild type and in Pax6 loss-of-function mutant Small eye mice (Sey allele).

Mapping the expression domains of Pax6, Dlxl and Otx2 on successive slices in the E12.5 dpc mouse brain revealed that these domains consistently respect some transverse and longitudinal boundaries as proposed in the prosomeric model (Puelles and Rubenstein, 1993). Here we present evidence that the previously identified two expression domains for $D l x l$ (Bulfone et al., 1993) abut upon regions (supraoptic paraventricular area, anterior hypothalamus, posterior preoptic area) that are specifically patterned by Pax6. Thus, both genes are specifying longitudinal expression boundaries and zones within the secondary prosencephalon.

According to the neuromeric theory (Bergquist and Källén, 1954; Coggeshall, 1964; Keyser, 1972; Kuhlenbeck, 1973), the developing neuroepithelium is subdivided into transverse compartments. Heterochronically proliferating histogenetic fields, which produce clusters of postmitotic neurons that move into the mantle zone, have been observed (Puelles et al., 1987; Wilson et al., 1990). It is assumed that delineation of a histogenetic field results from patterning effects of morphogenetic signals along the anteroposterior and dorsoventral axis in the undifferentiated neuroepithelium, while patterning along the mediolateral axis possibly leads to the migration of young neurons from deep to superficial mantle layers (Puelles, 1995). Accordingly, it is expected that sets of regulatory genes specifically expressed in individual histogenetic fields could be correlated with the fate-specification of these fields (Rubenstein and Puelles, 1994). The assignment of a histogenetic field to an adult structure is a difficult task because of the complex morphogenetic movements of differentiating neurons during development. Therefore, in this study, we initially followed the patterning of the developing wild-type diencephalon from stage E12.5 to stage E18.5 dpc. This allowed us to relate the specific patterning of already differentiated derivatives of ventral thalamus and hypothalamus in the prenatal brain to presumed neuromeric histogenetic fields of their origin.

At stage E12.5 dpc, the expression domains of Pax6, Dlxl and $O t x 2$ overlap extensively within the prosomere 3 (ventral thalamus). However, Dlxl is not detected within the dorsalmost domain of the ventral thalamus (where Pax6 and Otx2 are expressed); it is more abundantly expressed within its central region (see also Price et al., 1991). Our present analysis indicates that this field (according to its specific labelling by $D l x 1)$ later develops into the region of the differentiating zona incerta and reticular thalamic nucleus (E14.5 dpc) while, in the prenatal brain, it is related only to the rostralmost areas of these structures. Within the caudal part of prosomere 3, the expression domains of Pax6, Dlx 1 and Otx2 are grossly overlapping, a feature also observed in the caudal regions of zona incerta and ventral geniculate body at later developmental stages. A possible explanation (see Fig. 7) for the differential patterning of the derivatives of the ventral thalamus (zona incerta, reticular thalamic nucleus and ventral lateral geniculate body) is that they originate from distinct histogenetic fields within the territory of the ventral thalamus that are differently patterned by the transcription factors studied. This assumption is consistent with the results of a detailed ontogenetic study on the development of the diencephalon in Chinese hamster (Keyser, 1972, see also Fig. 7). Within the ventral thalamus, Keyser (1972) described two matrix primordia: (a) a pri- 
mordium that further develops into the rostral portion of the zona incerta, which appears to be specifically labelled only by Dlxl; (b) a primordium with two parts giving rise either to the ventral geniculate bodies, where Pax 6 and $D l x 1$ show nonoverlapping expression rostrally, or to the caudal portion of the zona incerta, where an extensive overlapping of the expression domains of both genes is observed.

Similar to the observed differential patterning of prosomere 3 (ventral thalamus) by Pax 6 and $D l x 1$, the expression of the two markers delineate neighbouring fields within prosomere 4 , representing the presumptive field of the entopeduncular nucleus (for Pax6) and a roundish structure within the lateral hypothalamus (for $D l x l$ ). Hypothalamic structures with similar characteristics have been reported to be derived from a common matrix of the forebrain (Marchard et al., 1986). The early developmental patterning of the region of the supraoptic paraventricular area by Pax 6 and $D l x l$ is maintained within the paraventricular nucleus at later developmental stages, suggesting its origin from this field. The field of hypothalamic cell cord, specifically labelled by $D l x l$ (prosomere 5) presumably gives rise to the anterodorsal hypothalamic nuclei.

In conclusion, the results from our expression analysis suggest that early confinement of the transcription factors Pax6 and $D l x l$ to distinct neuromeric fields is consistent with their fate specification in producing specific forebrain structures.

\section{Pax6 function is required to establish longitudinal boundaries along the dorsoventral axis in the secondary prosencephalon}

To examine the fidelity of the regional specification, a number of markers (Dlx1, Otx1, Otx2, Emx1, Emx2, Wnt3, Wnt3a, Prox1, Mash1 and Pax6) for distinct forebrain regions have been applied to Small eye brain sections. In general, these markers show normal distribution along the anteroposterior brain axis except for a lack of the normal ring-like expression of $0 t x 2$ at the level of the zona limitans intrathalamica. In addition, we detected an abnormal diffuse expression of Pax6 at the border between pretectum and midbrain (data not shown), possibly reflecting the underdevelopment of the tract of the posterior commissure. In contrast, the establishment of several longitudinal expression boundaries (e.g. between neocortex and lateral ganglionic eminence; anterior and posterior preoptic area; posterior preoptic area and suprachiasmatic area; zona limitans intrathalamica plus anterior hypothalamus and hypothalamic cell cord) that outline different strata within the secondary prosencephalon along the dorsoventral axis is compromised in the mutant brain and correlates with pattern and morphogenetic abnormalities. Similarly, changes in neuronal patterning have been reported in the forebrain of the zebrafish mutant cyclops as a consequence of the disruption of expression boundaries (Macdonald et al., 1994).

The disrupted patterning at the border between anterior and posterior preoptic area as seen in Sey/Sey is of special interest in relation to the eye phenotype. The neuroepithelium of the posterior preoptic area is supposed to contain progenitors destined to populate the optic stalk (Altman and Bayer, 1995). In the Sey/Sey diencephalon, the restrictive capacity of the border between anterior and posterior preoptic area is disrupted and the posterior preoptic area starts to express Dlxl abundantly, which might contribute to growth disturbances of the optic stalk in the mutant brain (Grindley et al., 1995; Schmahl et al., 1993).

The formation of the boundary between neocortex and lateral ganglionic eminence is a result of the earliest regionalisation of the forebrain, its subdivision into telencephalic cortex and basal ganglia. Previous expression analyses demonstrated that $D l x l$ and $D l x 2$ expression domains overlap, they are mainly confined to the subventricular zone of the basal telencephalon, establishing a sharp expression limit at the border between the striatum and the cortex (Bulfone et al., 1993, 1995; Price et al., 1991; also this study). In contrast, Pax6 is expressed in the ventricular zone of the cortex but not in the medial and lateral ganglionic eminence (Stoykova and Gruss, 1994; Walther and Gruss, 1991; also this study). In addition to Pax6 and Dlxl, several other genes respect the boundary between neocortex and lateral ganglionic eminence: Otx1, Otx2 (Simeone et al., 1992a,b); Emx2 (Simeone et al., 1993) and Tbr-1 (Bulfone et al., 1995). In Sey/Sey, in absence of Pax6 function, the restrictive capacity of this border is disturbed as early as at stage E12.5 dpc, as later indicated by a progressive extension of the $D l x l$-positive domain dorsally within the cortex. If $D l x l$-positive cells within the ventricular zone and subventricular zone of the medial and lateral ganglionic eminence are the 'striatal precursors,' then their progressive spreading into the cortex of the mutant brain may explain the hypocellularity of the striatum (where Pax6 is not expressed), a puzzling feature detected also in the $S e y^{\text {neu }}$ allele (Schmahl et al., 1993) and in human homozygous aniridia (Glaser et al., 1994). In addition, it might contribute to the establishment of the cortical phenotype.

The lack of the restrictive capacity at the border between neocortex and lateral ganglionic eminence in Sey/Sey may be explained by a loss of adhesive barrier properties in the Pax6 mutant. As reported by Fishell et al. (1993), in normal brain, DiI-labelled cells of the cortical ventricular zone cannot migrate within the ventricular zone of the lateral ganglionic eminence, implicating the existence of specific, adhesive properties at the border between neocortex and lateral ganglionic eminence. Similarly, adhesive differences between cortical and striatal cells have been recently demonstrated (Götz et al., 1996). These adhesive differences decline at later developmental stages, correlating with the previously reported decreased abundance of Pax6 transcripts in the cortical region after E15.5 dpc (Walther and Gruss, 1991). The close correlation between the region-specific expression of adhesion molecules such as R-cadherin (Matsunami and Takeichi, 1995; reviewed in Redies, 1995) or a selectinligand (Götz et al., 1996) in the forebrain with the Pax6 expression domains further supports a link between adhesive events and the patterning by Pax6. We are presently investigating this possibility.

\section{The Small eye forebrain phenotype and the function of Pax6}

The malformations in the telencephalic cortex of the Small eye brain as described in this study (Sey allele) are essentially the same as those reported for the Sey ${ }^{\text {neu }}$ allele (Schmahl et al., 1993) and for an aniridia homozygote in human (Glaser et al., 1994). These defects are consistent with the distribution of Pax6 transcripts in the ventricular zone of the developing telencephalon (Walther and Gruss, 1991); they have been suggested 
to reflect an abnormal migration of neuronal precursors in the cortical plate (Schmahl et al., 1993). While no severe pattern and morphogenetic disturbances of the diencephalon were reported for Sey ${ }^{\text {neu }}$ (Schmahl et al., 1993), the Sey allele shows severe alterations as described in this study. The basis for these differences most likely lies in the respective mutation. The point mutation in the Pax6 gene in Sey mice introduces a premature translation stop codon, truncating the protein between the paired and the paired type homeodomain (Hill et al., 1991). The Seyeu allele yields a 116-nucleotide insert within the Pax6 mRNA (as a consequence of a point mutation within a splice acceptor sequence), creating a nonsense mutation and truncating the protein carboxyterminal from the homeodomain (Hill et al., 1991), thereby possibly maintaining some residual function of Pax6.

Our analysis revealed that Pax6 function is required for the normal development of a number of forebrain regions (see Fig. 7) where it is specifically expressed and largely confined to the ventricular zone. At E12.5 dpc, the region corresponding to eminentia thalami (and the dorsal part of the ventral thalamus) shows abortive growth in the wrong laterocaudal direction. The derivative of the eminentia thalami is not detectable in the mutant brain (data not shown). As deduced from the Pax6 expression in Sey/Sey, supraoptic paraventricular area, anterior hypothalamus and posterior preoptic area are present, but the restricted expression of $D l x l$ (normally not expressed in these regions) seems to be lost. Later in development a paucity of tissue is detected within these regions (corresponding mainly to prosomere 3 and 4), which suggests defects in proliferation and/or patterning. Additional BrdU-labelling studies will help to elucidate this issue.

In the ventral thalamus, in addition to the ventricular zone, Pax6 is expressed also in the mantle zone. In the mutant brain, we detected severe abnormalities in patterning and specification of derivatives of the ventral thalamus. In Sey/Sey mice, progenitors assumed to populate different diencephalic compartments (dorsal and ventral thalamus) appear to be born in the neuroepithelium and express the corresponding markers. However, they fail to form distinctly patterned histogenetic fields at an early developmental stage and corresponding derivatives later on. This strongly suggests that downstream targets of the transcription factor Pax6 may ensure restriction of cell mixing but also segregation, assembly and migration of differently patterned forebrain progenitors (Fishell et al., 1993; reviewed in Redies, 1995).

The spatiotemporal expression of Pax6 in the developing forebrain is complex and we are only beginning to understand the logic behind it. It is obvious that $\operatorname{Pax} 6$ has numerous different functions in eye, nose and brain development. The patterning and specification of distinct forebrain structures, as shown in this study, are a new facet of the important role of Pax6 in vertebrate development.

We thank M. Price for the Dlx1 probe, A. Simeone for the Otx2 and Emx 2 probes, P. Salinas for the Wnt3 and Wnt $3 a$ probe and F. Guillemot for the Mash1 probe. We thank G. Oliver for assistance in the initial stage of this work. The technical assistance of $\mathrm{Ch}$. Müller and the photographic work of R. Altschäffel is highly acknowledged. We thank M. Kessel, G. Oliver and E. Stuart for the valuable suggestions and discussion on the manuscript. This work is supported by the Max-Plank-Gesellschaft. R. F. was supported by a Boehringer Ingelheim Fonds fellowship.

\section{REFERENCES}

Altman, J. and Bayer, S. A. (1995). Atlas of Prenatal Rat Brain Development. Boca Raton: CRC Press.

Alvarez-Bonaldo, G., Rosenfeld, M. G. and Swanson, L. W. (1995). Model of forebrain regionalization based onspatiotemporal patterns of POU-III homeobox gene expression, birthdates, and morphological features. J. Comp. Neurol. 355, 237-295.

Bergquist, H. and Källén, B. (1954). Notes on the early histogenesis and morphogenesis of the central nervous system in vertebrates. J. Comp. Neurol. 100, 627-660.

Boncinelli, E. (1994). Early CNS development: distal-less related genes and forebrain development. Curr. Opin. Neurobiol. 4, 29-36.

Bopp, D., Burri, M., Baumgartner, S., Frigerio, G. and Noll, M. (1986). Conservation of a large protein domain in the segmentation gene paired and in functionally related genes of Drosophila. Cell 47, 1033-1040.

Bulfone, A., Puelles, L., Porteus, M. H., Frohman, M. A., Martin, G. R. and Rubenstein, J. L. R. (1993). Spatially restricted expression of Dlx-1, Dlx-2 (Tes-1), Gbx-2, and Wnt-3 in the embryonic day 12.5 mouse forebrain defines potential transverse and longitudinal segmental boundaries. J. Neurosci. 13, 3155-3172.

Bulfone, A., Smiga, S. M., Shimamura, K., Peterson, A., Puelles, L. and Rubenstein, J. L. R. (1995). T-Brain-1: a homolog of Brachyury whose expression defines molecularly distinct domains within the cerebral cortex. Neuron 15, 63-78

Chalepakis, G., Stoykova, A., Wijnholds, J., Tremblay, P. and Gruss, P. (1993). Pax: Gene regulators in the developing nervous sxstem. J. Neurobiol. 24, 1367-1384.

Chisholm, A. D. and Horvitz, H. R. (1995). Patterning of the Caenorhabditis elegans head region by the Pax-6 family member vab- $b$. Nature 376, 52-55.

Coggeshall, R. E. (1964). A study of diencephalic development of the albino rat. J. comp. Neurol. 122, 241-269.

Dressler, G. R., Deutsch, U., Balling, R., Simon, D., Guénet, J.-L. and Gruss, P. (1988). Murine genes with homology to Drosophila segmentation genes. Development 104 (Supplement), 181-186.

Figdor, M. C. and Stern, C. D. (1993). Segmental organization of embryonic diencephalon. Nature 363, 630-634.

Fishell, G., Manson, C. A. and Hatten, M. E. (1993). Dispersion of neural progenitors within the germinal zones of the forebrain. Nature 362, 636-638.

Fujiwara, M., Uchida, T., Osumi-Yamashita, N. and Eto, K. (1994). Uchida rat $(r S e y)$ : a new mutant rat with craniofacial abnormalities resembling those of the mouse Sey mutant. Differentiation 57, 31-38.

Glaser, T., Jepeal, L., Edwards, J. G., Young, S. R., Favor, J. and Maas, R. L. (1994). PAX6 gene dosage effect in a family with congenital cataracts, aniridia, anophthalamia and central nervous system defects. Nature Genet. 7, 463-471.

Götz, M., Wizenmann, A., Reinhard, S., Lumsden, A. and Price, J. (1996). Selective adhesion of cells from different telencephalic regions. Neuron 16, 551-564.

Grindley, J. C., Davidson, D. R. and Hill, R. E. (1995). The role of Pax-6 in eye and nasal development. Development 121, 1433-1442.

Guillemot, F. and Joyner, A. L. (1993). Dynamic expression of the murine Achaete-Scute homologue Mash-1 in the developing nervous system. Mech. Dev. 42, 171-185.

Guthrie, S. (1996). Patterning the hindbrain. Curr. Opin. Neurobiol. 6, 41-48.

Halder, G., Callaerts, P. and Gehring, W. J. (1995). Induction of ectopic eyes by targeted expression of the eyeless gene in Drosophila. Science 267, 17881792.

Hanson, I. M., Fletcher, J. M., Jordan, T., Brown, A., Taylor, D., Adams, R. J., Punnet, H. H. and van Heyningen, V. (1994). Mutations at the PAX6 locus are found in heterogeneous anterior segment malformations including Peters' anomaly. Nature Genet. 6, 168-173.

Hatini, V., Tao, W. and Lai, E. (1994). Expression of winged helix genes BF1 and $B F-2$ defines adjacent domains within the developing forebrain and retina. J. Neurobiol. 25, 1293-1309.

Hill, R. E., Favor, J., Hogan, B. L. M., Ton, C. C. T., Saunders, G. F., Hanson, I. M., Prosser, J., Jordan, T., Hastie, N. D. and van Heyningen, V. (1991). Mouse Small eye results from mutations in a paired-like homeobox-containing gene. Nature 354, 522-525.

Hogan, B. L. M., Hirst, E. M. A., Horsburgh, G. and Hetherington, C. M. (1988). Small eye (Sey): a mouse model for the genetic analysis of cranofacial abnormalities. Development 103 Supplement, 115-119.

Hogan, B. L. M., Horsburgh, G., Cohen, J., Hetherington, C. M., Fisher, G. and Lyon, M. F. (1986). Small eyes (Sey): a homozygous lethal mutation on 
chromosome 2 which affects the differentiation of both lens and nasal placodes in the mouse. J. Embryol. Exp. Morph. 97, 95-110.

Jordan, T., Hanson, I., Zaletayev, D., Hodgson, S., Prosser, J., Seawright, A., Hastie, N. and van Heyningen, V. (1992). The human PAX6 gene is mutated in two patients with aniridia. Nature Genet. 1, 328-332.

Keyser, A. (1972). The development of the diencephalon of the chinese hamster. An investigation of the validity of the criteria of subdivision of the brain. Acta Anat. 83 (Suppl. 59), 1-178.

Krumlauf, R. (1994). Hox genes in vertebrate development. Cell 78, 191-201.

Kuhlenbeck, H. (1973). The Central Nervous System of Vertebrates Basel Karger.

Lumsden, A. (1990). The development and significance of hindbrain segmentation. Sem. Dev. Biol. 1, 117-125.

Macdonald, R., Xu, Q., Barth, K. A., Mikkola, I., Holder, N., Fjose, A., Krauss, S. and Wilson, S. W. (1994). Regulatory gene expression boundaries demarcate sites of neuronal differentiation in the embryonic zebrafish forebrain. Neuron 13, 1039-1053.

Mansouri, A., Stoykova, A. and Gruss, P. (1994). Pax genes in development. J. Cell Sci. Supplement 18, 35-42.

Marchard, R., Lajoie, L. and Blanchet, C. (1986). Histogenesis at the level of the basal forebrain: the entopeduncular nucleus. Neurosci. 17, 591-607.

Mastick, G. S. and Easter, S. S. J. (1996). Initial organization of neurons and tracts in the embryonic mouse fore- and midbrain. Dev. Biol. 173, 79-94.

Matsunami, H. and Takeichi, M. (1995). Fetal brain subdivisions defined by R- and E-cadherin expressions: evidence for the role of cadherin activity in region-specific, cell-cell adhesion. Dev. Biol. 172, 466-478.

Matsuo, T., Osumi-Yamashita, N., Noji, S., Ohuchi, H., Koyama, E., Myokai, F., Matsuo, N., Taniguchi, S., Doi, H., Ninomiya, Y., Fujiwara, M., Watanabe, T. and Eto, K. (1993). A mutation in the Pax-6 gene in rat small eye is associated with impaired migration of midbrain crest cells Nature Genet. 3, 299-304.

McMahon, A. P. (1992). A superfamily of putative developmental signalling molecules related to the proto-oncogene Wnt-1/int-1. In Advances in Developmental Biology (ed. Wassarman, P. M.) pp. 31-60.

Nusse, R. and Varmus, H. E. (1992). Wnt genes. Cell 69, 1073-1087.

Oliver, G., Sosa-Pineda, B., Geisendorf, S., Spana, E. P., Doe, C. Q. and Gruss, P. (1993). Prox 1, a prospero-related homeobox gene expressed during mouse development. Mech. Dev. 44, 3-16.

Paxinos, G., Törk, I., Tecott, L. H. and Valentino, K. L. (1991). Atlas of the Developing Rat Brain. San Diego: Academic Press.

Porteus, M. H., Bulfone, A., Ciaranello, R. D. and Rubenstein, J. L. (1991) Isolation and characterization of a novel cDNA clone encoding a homeodomain that is developmentally regulated in the ventral forebrain. Neuron 7, 221-229.

Price, M., Lazzaro, D., Pohl, T., Mattei, M.-G., Rüther, U., Olivo, J.-C. Duboule, D. and Di Lauro, R. (1992). Regional expression of the homeobox gene $N k x-2.2$ in the developing mam malian forebrain. Neuron $\mathbf{8}$, 241-255.

Price, M., Lemaistre, M., Pischetola, M., Di Lauro, R. and Duboule, D. (1991). A mouse gene related to Distal-less shows a restricted expression in the developing forebrain. Nature 351, 748-751.

Puelles, L. (1995). A segmental morphological paradigm for understanding vertebrate forebrains. Brain Behav. Evol. 46, 319-337.

Puelles, L., Amat, J. A. and Martinez-de-la-Torre, M. (1987). Segmentrelated, mosaic neurogenetic pattern in the forebrain and mesencephalon of early chick embryos: I. Topography of AChE-positive neuroblasts up to stage HH18. J. Comp. Neurol. 266, 247-268.

Puelles, L. and Rubenstein, J. L. R. (1993). Expression patterns of homeobox and other putatitive regulatory genes in the embryonic mouse forebrain suggests a neuromeric organization. Trends Neurosci. 16, 472-479.

Quiring, R., Walldorf, U., Kloter, U. and Gehring, W. J. (1994). Homology of the eyeless gene of Drosophila to the Small eye gene in mice and Aniridia in humans. Science 265, 785-789.
Redies, C. (1995) Cadherin expression in the developing vertebrate CNS: from neuromeres to brain nuclei and neural circuits. Exp. Cell Res. 220, 243-256.

Roberts, R. C. (1967). Small-eyes, a new dominant mutant in the mouse. Genet. Res. 9, 121-122.

Roelink, H. and Nusse, R. (1991). Expression of two members of the Wnt family during mouse development-restricted temporal and spatial patterns in the developing neural tube. Genes Dev. 5, 381-388.

Rosenfeld, M. G. (1991). POU-domain transcription factors: POU-er-full developmental regulators. Genes Dev. 5, 897-907.

Rubenstein, J. L. R. and Puelles, L. (1994). Homeobox gene expression during development of the vertebrate brain. Curr. Top. Dev. Biol. 29, 1-63.

Salinas, P. C. and Nusse, R. (1992). Regional expression of the Wnt-3 gene in the developing mouse forebrain in relationship to diencephalic neuromeres. Mech. Dev. 39, 151-160.

Schmahl, W., Knoedlseder, M., Favor, J. and Davidson, D. (1993). Defects of neuronal migratzion and the pathogenesis of cortical malformations are associated with small eye (sey) in the mouse, a point mutation at the Pax-6 locus. Acta Neuropathol. 86, 126-135.

Shimamura, K., Hartigan, D. J., Martinez, S., Puelles, L. and Rubenstein, J. L. R. (1995). Longitudinal organization of the anterior neural plate and neural tube. Development 121, 3923-3933.

Simeone, A., Acampora, D., Gulisano, M., Stornaiuolo, A. and Boncinelli, E. (1992a). Nested expression domains of four homeobox genes in developing rostral brain. Nature 358, 687-690.

Simeone, A., Acampora, D., Mallamaci, A., Stornaiuolo, A., D'Apice, M. R., Nigro, V. and Boncinelli, E. (1993). A vertebrate gene related to orthodenticle contains a homeodomain of the bicoid class and demarcates anterior neuroectoderm in the gastrulating mouse embryo. EMBO J. 12, 2735-2747.

Simeone, A., Gulisano, M., Acampora, D., Stornaiuolo, A., Rambaldi, M. and Boncinelli, E. (1992b). Two vertebrate homeobox genes related to the Drosophila empty spiracles gene are expressed in the embryonic cerebral cortex. EMBO J. 11, 2541-2550.

Stoykova, A. and Gruss, P. (1994). Roles of Pax-genes in developing and adult brain as suggested by expression patterns. J. Neurosci. 14, 1395-1412.

Timsit, S., Martinez, S., Allinquant, B., Peyron, F., Puelles, L. and Zalc, B. (1995). Oligodendrocytes originate in a restricted zone of the embryonic ventral neural tube defined by DM-20 mRNA expression. J. Neurosci. 15 1012-1024.

Tole, S. and Patterson, P. H. (1995). Regionalization of the developing forebrain - a comparison of FORSE-1, Dlx-2, and BF-1. J. Neurosci. 15, 970-980.

Ton, C. C. T., Hirvonen, H., Miwa, H., Weil, M. M., Monaghan, P., Jordan, T., van Heyningen, V., Hastie, N. D., Meijers-Heijboer, H., Drechsler, M., Royer-Pokora, B., Collins, F., Swaroop, A., Strong, L. C. and Saunders, G. F. (1991). Positional cloning of a paired box- and homeoboxcontaining gene from the Aniridia region. Cell 67, 1059-1074.

Wallin, J., Mizutani, Y., Imai, K., Miyashita, N., Moriwaki, K., Taniguchi, M., Koseki, H. and Balling, R. (1993). A new Pax gene, Pax-9, maps to mouse chromosome 12. Mammal. Genome 4, 354-358.

Walther, C. and Gruss, P. (1991). Pax-6, a murine paired box gene, is expressed in the developing CNS. Development 113, 1435-1449.

Walther, C., Guénet, J.-L., Simon, D., Deutsch, U., Jostes, B., Goulding M., Plachov, D., Balling, R. and Gruss, P. (1991). Pax: a murine multigene family of paired box containing genes. Genomics 11, 424-434.

Wilson, S. W., Ross, L. S., Parrett, T. and Easter, S. S. J. (1990). The development of a simple scaffold of axon tracts in the brain of the embryonic zebrafish, Brachydanio rerio. Development 108, 121-145.

Zhang, Y. and Emmons, S. W. (1995). Specification of sense organ identity by a Caenorhabditis elegans Pax-6 homologue. Nature 376, 55-59.

(Accepted 13 August 1996) 